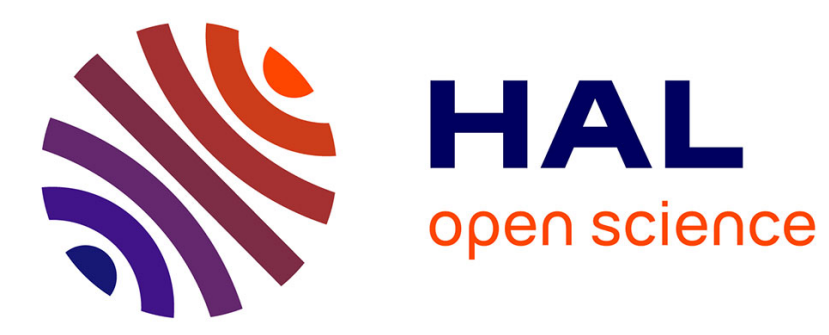

\title{
Blind Digital Modulation Classification based on M-TH Power Nonlinear Transformation
}

Vincent Gouldieff, Jacques Palicot, Steredenn Daumont

\section{To cite this version:}

Vincent Gouldieff, Jacques Palicot, Steredenn Daumont. Blind Digital Modulation Classification based on M-TH Power Nonlinear Transformation. 2016 IEEE Global Conference on Signal and Information Processing (GlobalSIP), Dec 2016, Washington DC, United States. 10.1109/GlobalSIP.2016.7905922 . hal-01396252

\section{HAL Id: hal-01396252 \\ https://hal.science/hal-01396252}

Submitted on 14 Nov 2016

HAL is a multi-disciplinary open access archive for the deposit and dissemination of scientific research documents, whether they are published or not. The documents may come from teaching and research institutions in France or abroad, or from public or private research centers.
L'archive ouverte pluridisciplinaire HAL, est destinée au dépôt et à la diffusion de documents scientifiques de niveau recherche, publiés ou non, émanant des établissements d'enseignement et de recherche français ou étrangers, des laboratoires publics ou privés. 


\title{
BLIND DIGITAL MODULATION CLASSIFICATION BASED ON M-TH POWER NONLINEAR TRANSFORMATION
}

\author{
Vincent Gouldieff ${ }^{1}$, Jacques Palicot ${ }^{1}$, Steredenn Daumont ${ }^{2}$ \\ ${ }^{1}$ CentraleSupelec/IETR, Avenue de la Boulaie - CS 47601, 35576 Cesson-Sevigne Cedex, France \\ ${ }^{2}$ Zodiac Data Systems, 2 Rue de Caen, 14740 Bretteville-l'Orgueilleuse, France \\ Email: \{vincent.gouldieff, jacques.palicot\}@ supelec.fr, steredenn.daumont@ zodiacaerospace.com
}

\begin{abstract}
Automatic Modulation Classification (AMC) has received a major attention last decades, as a required step between signal detection and demodulation. In the fully-blind scenario, this task turns out to be quite challenging, especially when the computational complexity and the robustness to uncertainty matter. AMC commonly relies on a preprocessor whose function is to estimate unknown parameters, filter the received signal and sample it in a suitable way. Any preprocessing error inherently leads to a performance loss. To improve the robustness of the blind AMC, we propose to proceed almost directly on the received signal - with neither matched-filtering step nor synchronization step. In this paper, Analytical $M^{t h}$-Power nonlinear Transformation (AMPT) is considered for its robustness towards timing, phase and frequency uncertainty. The generated feature-vector then feeds a Minimum Distance classifier. Numerical simulations show the effectiveness of the proposed method for a 7-class problem of low-order modulations.
\end{abstract}

Index Terms - Automatic Modulation Classification, $M^{t h}$ Power nonlinear Transform, Blind Detection.

\section{INTRODUCTION}

The demand for reconfigurable radio technologies - such as Cognitive Radio (CR) - has led to an increasing need for blind signal processing, such as spectrum sensing [1], standard recognition [2] and feature detection [3]. Since the early 90', Automatic Modulation Classification (AMC) has been extensively studied as it represents a key challenge in Cognitive Radio. In recent years, several techniques for AMC have been reported in the literature [4]. Most of these methods rely on a two-steps architecture: a signal preprocessing task followed by a recognition algorithm (see e.g., Fig. 1).

The first step generally consists of a demodulator which feeds the recognition algorithm with a filtered, normalized and symbolspaced sampled signal. Therefore, numerous inherent parameters of the received signal - such as the symbol rate, pulse shaping, carrier frequency, signal and noise powers - are generally assumed to be perfectly known at the receiver. This strong assumption is in contradiction with the blind property needed in a smart receiver.

The second step (the modulation recognition algorithm itself) may rely on a Likelihood-Based (LB) or a Feature-Based (FB) method [4]. While the former is optimal in the Correct Classification Rate (CCR) sense, the latter is often favoured for its near-optimal performance at a lower complexity. As a consequence, we propose in the scope of this article a new Feature-Based classifier. Obviously, the quality of the whole blind algorithm depends on both the performance of the preprocessor and of the recognition algorithm.

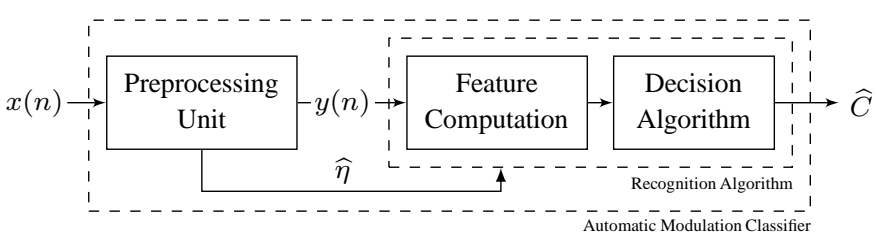

Fig. 1. A common Feature-Based AMC architecture

FB classifiers rely on two sub-steps, namely a feature computation step and a decision making step. Signal statistics-based features, for instance Higher Order Statistics (HOS), have been widely studied for their ability to differentiate between digital modulations. Cyclostationarity is also a good candidate in the blind scenario for its robustness to frequency, phase and time offsets [5]. Numerous decision algorithms were proposed, as Artificial Neural Network (ANN), Support Vector Machine (SVM) or Decision Tree (DT) [5][6].

Swami et al. proposed a classifier relying on Higher Order $\mathrm{Cu}-$ mulants (HOCs) and DT to recognize PAM, QAM and PSK modulations [7]: this method is indeed efficient but unsuited in the blind scenario. As a step towards blindness, some improved HOS-based methods were proposed to face unknown signal power [8] or carrier frequency offset [9][10]. As expected, the CCR is improved with these methods when the preprocessing task is assumed non-ideal. Cyclostationarity was also proposed to detect spectrum holes [3] and classify analog and digital modulations [11]. Its main drawback is the complexity entailed by the computation of the cyclic function.

Recently, several fully blind AMC techniques based on the $M^{t h}$-Power nonlinear Transformation (MPT) were suggested $[12,13,14]$. MPT was first studied as a tool for carrier and timing synchronization [15] and then used for AMC in the 90' [16]. MPT is of major interest since the produced features are insensitive to time, frequency and phase offsets: thus, no elaborate preprocessing step is needed. In most of the previous work, the recognition only relies on the number of peaks generated by the $M \mathrm{PT}$ for different $M$. Then, the classifier is unsuited for an efficient classification if the test-set contains some constellations of the same order.

We propose a novel fully-blind method for AMC, based on the theoretical power of the peaks generated by the MPTs. This technique - we called Analytical MPT (AMPT) - is suitable for PSK, PAM, QAM and hybrid modulations. Moreover, the preprocessing task is the simplest possible to improve the robustness of the whole classifier to estimation uncertainty. A simple MD classifier is considered for a better fusion of the different powers in the feature-space.

The paper is organized as follows: In Section 2 the problem under study is formulated, while in Section 3 the proposed blind AMC method is described. In Section 4 the theoretical development of the references is carried out. The performance is studied via extensive simulations in Section 5. 


\section{PROBLEM FORMULATION}

Let us assume that the signal-of-interest consists of a pseudobaseband pulse-shaped digital signal in AWGN channel. This scenario is relevant, for instance, for fixed satellite communication systems. The signal is then modelled as

$$
x(n)=a \cdot e^{i \cdot\left(2 \pi f_{r} \cdot n+\phi\right)} \cdot \sum_{k} s(k) \cdot h\left(n T_{e}-k T-\tau\right)+\omega(n)
$$

where $a$ is the amplitude of the signal, $f_{r}$ is the residual carrier frequency offset, $\phi$ is the initial phase, $s(k)$ is the unknown sequence of i.i.d. symbols, $h$ is the pulse shaping function, $T_{e}$ is the sampling period, $T$ is the symbol period, $\tau$ is the initial delay and $\omega$ is the sampled and unfiltered white Gaussian noise.

We consider that $h$ is a root raised cosine (RRC) filter with unknown roll-off $\beta$ and that the oversampling factor $\rho=\frac{T}{T_{e}}$ respects the Shannon's theorem i.e., $\rho \geq 1+\beta$. Remark that we do not assume any time or frequency synchronization here. We denote by $N$ the number of considered symbols. Thus, we dispose of $N_{e}=\rho N$ samples for the classification. We assume that all the parameters in (1) are constant on $\left[0 ; N_{e}-1\right]$.

We consider the 7-class problem of $\mathcal{C}=\{$ BPSK, QPSK, 8PSK, 8AMPM, R8QAM, C8QAM, 16QAM $\}$ in order to show the effectiveness of the proposed method on quite similar zero-mean and unitvariance constellations (see Fig. 2).

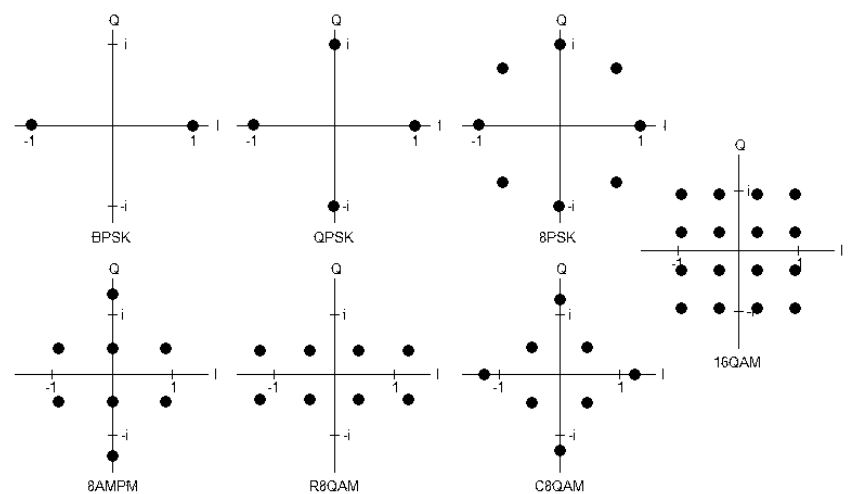

Fig. 2. Representation of the considered zero-mean and unitvariance constellations in the complex plan

\section{DESCRIPTION OF THE PROPOSED AMC METHOD}

\subsection{Basic definitions}

In the scope of the paper, we define the MPT of $x(n)$ as the $M^{t h}$ root of the Power Spectral Density (PSD) of $x^{M}(n)$

$$
\operatorname{MPT}_{x}(f)=N_{s}-\frac{2}{M} \cdot\left|\sum_{n=0}^{N_{e}-1} x^{M}(n) \cdot e^{-2 i \pi n f}\right|^{\frac{2}{M}}
$$

This transform may show spectral lines around the null frequency for some values of $M$ : their existence and power mainly depend on the emitted constellation.

While other MPT-based techniques in the literature only exploit the existence property $[12,13,14,16]$ - and then fail to differentiate between quite similar constellations such as QPSK and 16QAM, or BPSK and R8QAM - we instead propose to exploit the power of the spectral lines as strong features. We then dispose of more useful information, which also further enables to derive a soft decision.

\subsection{Proposed preprocessing unit}

In the fully-blind scenario, some preprocessing tasks have to be carried out. First, a spectrum sensor (see e.g., [1]) followed by a standard recognizer (see e.g., [2]) respectively locate the bands where the AMC have to be performed and provide information for further detection stages. Thereby, these tasks are assumed correctly performed without affecting the blind property of the proposed method. Then, a simple normalizer and parameter estimator proceed as follows:

\subsubsection{Normalization}

Assume that no interferer is present in the $-3 d B$ bandwidth of the received signal, it is clear that $P_{3 d B}=P_{\omega} \cdot \rho^{-1}+P_{u} \cdot P_{H, 3 d B}$, where $P_{\omega}$ is the power of the noise, $P_{u}$ is the power of the noiseless signal-of-interest $u(n)=x(n)-\omega(n)$, and $P_{H, 3 d B}$ is the power of the pulse-shaping filter in its $-3 d B$ bandwidth. With a root-raised cosine filter, we have $P_{H, 3 d B}=1-\frac{\beta}{2}+\frac{\beta}{\pi}$ and we eventually get

$$
\widehat{P_{u}}=\frac{P_{3 d B}-\widehat{P_{\omega}} \cdot \widehat{\rho}^{-1}}{1-\frac{\widehat{\beta}}{2}+\frac{\widehat{\beta}}{\pi}}
$$

If not known, remark that $\beta, \rho$ and $P_{\omega}$ can be easily estimated using the formerly computed PSD of $x(n)$.

\subsubsection{Residual Carrier Frequency Estimation}

The residual carrier frequency $f_{r}$ also has to be estimated in order to locate the spectral lines - which occur at frequencies $f_{M}=M f_{r}$.

Such an estimation is generally performed by a maximum-tomean detector applied on the computed MPTs:

$$
M \widehat{f_{r}}=\underset{f, M}{\operatorname{argmax}}\left(M \mathrm{PT}_{x}(f)-\overline{M \mathrm{PT}_{x}(f)}\right)
$$

where $\overline{M \mathrm{PT}_{x}(f)}$ is the mean value of $\mathrm{MPT}_{x}(f)$ around the peak.

\subsubsection{Output of the preprocessor}

The preprocessor feeds the feature-computer with the normalized version of $x(n)$ and with the set of estimated parameters (Fig. 1):

$$
y(n)=x(n) \cdot{\widehat{P_{u}}}^{-\frac{1}{2}} \text { and } \widehat{\eta}=\left\{\widehat{\beta}, \widehat{\rho}, \widehat{P_{\omega}}\right\}
$$

In realistic conditions, band-pass pre-filtering is considered as well for a better rejection of out-of-band noise and adjacent signals.

\subsection{Decision Making via Minimum Distance classification}

We use a Minimum Distance (MD) classifier to fusion the features of set $\left\{M_{i} \mathrm{PT}_{y}\left(M_{i} \widehat{f}_{r}\right), M_{i} \in \mathcal{M}\right\}$ and compare them with the theoretical references. Denote by $\Pi$ the feature-vector

$$
\Pi=\left[M_{1} \mathrm{PT}_{y}\left(M_{1} \widehat{f}_{r}\right) \cdots M_{m} \mathrm{PT}_{y}\left(M_{m} \widehat{f}_{r}\right)\right]
$$

and by $\Pi_{t h}^{C}$ the theoretical feature-vector if $C$ is emitted (please refer to the next Section for the derivation of the references)

$$
\Pi_{t h}^{C}=\left[\begin{array}{lll}
M_{1} \mathrm{PT}_{t h}^{C} & \cdots & M_{m} \mathrm{PT}_{t h}^{C}
\end{array}\right]
$$

the distance is simply computed with norm-2 as

$$
\Delta^{C}=\left\|\Pi-\Pi_{t h}^{C}\right\|_{2}
$$

We finally get the estimated constellation $\widehat{C}$ as

$$
\widehat{C}=\underset{C \in \mathcal{C}}{\operatorname{argmin}} \Delta^{\mathcal{C}}
$$




\subsection{Description of the proposed algorithm}

The proposed fully-blind whole AMC algorithm is summed up in Fig. 3, where the general structure of Fig. 1 is conserved.

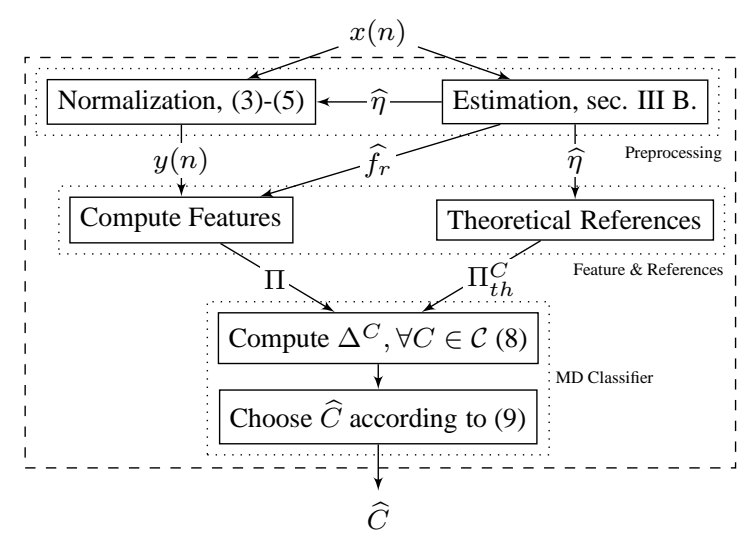

Fig. 3. The proposed fully-blind AMC algorithm

\section{THEORETICAL REFERENCES}

In this section, we derive the theoretical power of the spectral lines occurring in the MPT function. Since the PSD of a cyclostationary signal is insensitive to phase and time offsets [16], we assume that $\phi=0$ and $\tau=0$ in (1). Moreover, without loss of generality and for ease of notation, we assume that the residual frequency $f_{r}$ is null.

We first work on a noise-free, continuous-time and infinite length signal. The derivation of the references in realistic conditions is carried out in future work. In this section, we then consider:

$$
u(t)=\sum_{k} s(k) \cdot h(t-k T)
$$

where the notations were formerly defined in Section II.

For the sake of a better understanding, we derive the theoretical references when $M=2$ and $M=4$. References for other values of $M$ follow the exact same principle.

\subsection{Computation of $2 P T_{t h}^{C}$}

As developed in [15], $u^{2}$ easily splits into two sums

$$
u^{2}(t)=u_{20}(t)+u_{11}(t)
$$

where

$$
\begin{gathered}
u_{20}(t)=\sum_{k} s^{2}(k) \cdot h^{2}(t-k T) \\
u_{11}(t)=\sum_{k \neq k^{\prime}} s(k) \cdot s\left(k^{\prime}\right) \cdot h(t-k T) \cdot h\left(t-k^{\prime} T\right)
\end{gathered}
$$

The presence of a frequency peak in the PSD of $u^{2}(t)$ at null frequency is made obvious by applying the Poisson Summation Formula on fictive signal $u_{20}(t)$. In fact, we have

$$
\mathbb{E}\left[u_{20}(t)\right]=\frac{\mathbb{E}\left[s^{2}\right]}{T} \sum_{m} e^{i \cdot m \cdot t} \cdot H_{2}(m)
$$

where $m$ corresponds to the multiples of the symbol frequency and $H_{2}(f)$ corresponds to the Fourier Transform of $h^{2}(t)$. Note that $u_{11}(t)$ doesn't produce any spectral line for $i . i . d$. symbol sets since $\mathbb{E}[s(k) s(k+n)]=0$ whatever $n \neq 0$. In the scope of the paper, $h^{2}(t)$ corresponds to a raised cosine (RC) filter. Then $H_{2}(0)=T$ and the theoretical reference when $M=2$ is finally derived as:

$$
2 \mathrm{PT}_{t h}^{C}=\left|\mathbb{E}\left[s^{2}\right]\right|
$$

Theoretical values of $2 \mathrm{PT}_{t h}^{C}$ are tabulated as follows:

\begin{tabular}{|c|c|c|c|c|c|c|c|}
\hline $\mathrm{C} \rightarrow$ & BPSK & QPSK & 8PSK & 8AMPM & R8QAM & C8QAM & 16QAM \\
\hline \hline $2^{\text {PT }} C$ & 1 & 0 & 0 & 0.2 & $2 / 3$ & 0 & 0 \\
\hline
\end{tabular}

Table 1. Tabulated values of $2 \mathrm{PT}_{t h}^{C}$ for $C \in \mathcal{C}$.

\subsection{Computation of $4 P T_{t h}^{C}$}

The same development is carried out for the fourth power of $u(t)$, which is plainly developed as

$$
u^{4}(t)=u_{4000}(t)+u_{3100}(t)+u_{2200}(t)+u_{2110}(t)+u_{1111}(t)
$$

where the notation in the right-hand side terms is straightforward when compared to (11) - (13).

Now, both terms $u_{4000}(t)$ and $u_{2200}(t)$ have an impact on the power of the spectral lines while the remaining terms don't (they actually show null expectations). Then, following the method of IV. A and after some simplifications in the indices, we may get:

$$
4 \mathrm{PT}_{t h}^{C}=\left|\frac{1}{T}\left(\mathbb{E}\left[s^{4}\right] H_{4}(0)+6\left(\mathbb{E}\left[s^{2}\right]\right)^{2} \sum_{k>0} H_{22}^{(k)}(0)\right)\right|^{\frac{1}{2}}
$$

where $H_{4}(f)$ stands for the Fourier Transform of $h^{4}(t)$ and $H_{22}^{(k)}(f)$ stands for the Fourier Transform of $h^{2}(t) \cdot h^{2}(t-k T)$.

Note that no simple analytical formula can be found neither for $H_{4}(0)$ nor for $H_{22}^{(k)}(0)$ and that they depend on $\beta$. However, they can be easily computed numerically. A few values of $4 \mathrm{PT}_{t h}^{C}$ as a function of $\beta$ are gathered in Table 2 .

\begin{tabular}{|c|c|c|c|c|c|c|c|}
\hline$\beta \downarrow C$ & BPSK & QPSK & 8PSK & 8AMPM & R8QAM & C8QAM & 16QAM \\
\hline \hline 0.15 & 1.233 & 0.856 & 0.000 & 0.734 & 0.770 & 0.929 & 0.713 \\
\hline 0.25 & 1.200 & 0.881 & 0.000 & 0.754 & 0.743 & 0.961 & 0.737 \\
\hline 0.35 & 1.173 & 0.907 & 0.000 & 0.774 & 0.719 & 0.991 & 0.760 \\
\hline
\end{tabular}

Table 2. Tabulated values of $4 \mathrm{PT}_{t h}^{C}$ for $C \in \mathcal{C}$ and for different $\beta$.

\subsection{Representation in the feature-space}

Fig. 4 shows the theoretical and simulated feature-vectors in the feature-space. Note that a proper noise-corrective term is added to the references so that they better match the simulated features.

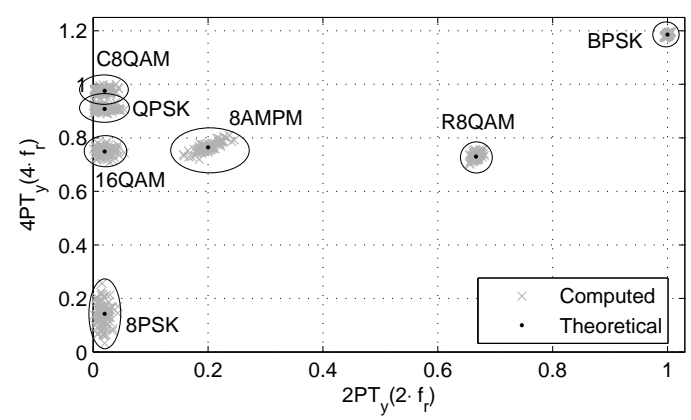

Fig. 4. Theoretical and simulated feature-vectors, for 100 realizations per constellation, $\mathrm{SNR}=15 \mathrm{~dB}, \beta=0.3, \rho=2$ and $N=1 e^{4}$

Obviously, some sets of constellations will be easier to distinguish than other sets, as Fig. 4 and Table 3 show. Note that Table 3 gives the distance between constellations $C$ and $C^{\prime}$ as $\Delta^{C, C^{\prime}}=$ $\left\|\Pi_{t h}^{C}-\Pi_{t h}^{C^{\prime}}\right\|_{2}$. In future work, it would be interesting to study the probabilistic behaviour of the features. Then, we could derive the theoretical Correct Classification Rate (CCR) of the proposed AMC method. Herein, we present numerical results in the next section. 


\begin{tabular}{|c|c|c|c|c|c|c|c|c|}
\hline & \multicolumn{7}{|c|}{ Constellation } \\
\hline & & BPSK & QPSK & 8PSK & 8AMPM & R8QAM & C8QAM & 16QAM \\
\hline \multirow{7}{*}{ 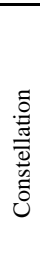 } & BPSK & 0 & 1.004 & 1.419 & 0.905 & 0.564 & 0.988 & 1.055 \\
\hline & QPSK & & 0 & 0.761 & 0.219 & 0.656 & 0.068 & 0.159 \\
\hline & $8 \mathrm{PSK}$ & & & 0 & 0.639 & 0.861 & 0.829 & 0.602 \\
\hline & 8AMPM & & & & 0 & 0.468 & 0.268 & 0.161 \\
\hline & \begin{tabular}{|l|} 
R8QAM \\
\end{tabular} & & & & & $\overline{0}$ & 0.677 & 0.629 \\
\hline & \begin{tabular}{|l|} 
C8QAM \\
\end{tabular} & & & & & & 0 & 0.227 \\
\hline & 16QAM & & & & & & & 0 \\
\hline
\end{tabular}

Table 3. Distance Matrix in the proposed feature-space

\section{NUMERICAL RESULTS}

Extensive simulations were carried out to show the effectiveness of the proposed method. The performance of our algorithm is also compared with the CCRs obtained with Swami's $C_{40}$-based classifier ([7], Ex. 1) in both the blind and the non-blind scenario. The experiments were performed on 10000 trials per constellation. Recall that $N$ is the number of symbols used for the classification.

\subsection{Performance of the proposed classifier}

Table 4 shows the Confusion Matrix (CM) for the proposed 7-class problem in the blind scenario. For a better reading, ". " means $0 \%$. As expected, the worst CCR is obtained for QPSK/C8QAM since these constellations are the closest in the 2PT/4PT feature-space.

Considering other features - such as higher-order or conjugated MPTs - would improve the distance between these constellations. However, increasing $M$ may also involve a degradation of the CCR at low SNRs since the features would show a more stochastic behaviour as $M$ grows and as the SNR weakens.

\begin{tabular}{|c|c|c|c|c|c|c|c|c|}
\hline \multicolumn{2}{c|}{} & \multicolumn{7}{c|}{ Transmitted Constellation } \\
\cline { 2 - 9 } \multicolumn{2}{c|}{} & BPSK & QPSK & 8PSK & 8AMPM & R8QAM & C8QAM & 16 QQM \\
\hline
\end{tabular}

Table 4. Confusion Matrix with $S N R=10 \mathrm{~dB}$ and $N=1000$ for the proposed 7-class problem and in the blind scenario

Fig. 5 shows the CCR in the blind scenario for the proposed 7class problem as a function of the SNR. In this context, we have set $N=1000$ and $\rho=2$. As expected, set $\{$ BPSK, R8QAM, 8PSK $\}$ is the easiest to classify, while the CCR for $\{$ QPSK, C8QAM $\}$ remains slightly lower for medium-to-high SNR (see especially Table 4).

\subsection{Performance comparison with the $C_{40}$-based classifier}

We compare the CCR obtained by Swami's $C_{40}$-based method in [7] - commonly used as a benchmark in the literature - with the proposed AMPT-based classifier, in the same conditions as Fig. 5.

Mean performances of the methods in both the blind and the non-blind scenario are depicted in Fig. 6. The A2PT/A4PT-based classifier shows a slight increase in the performance of the classification in the non-blind scenario. Besides, in the fully-blind scenario, the A2PT/A4PT-based classifier clearly outperforms Swami's method. This is mainly due to a better robustness of the proposed features to inherent estimation errors at the preprocessor.

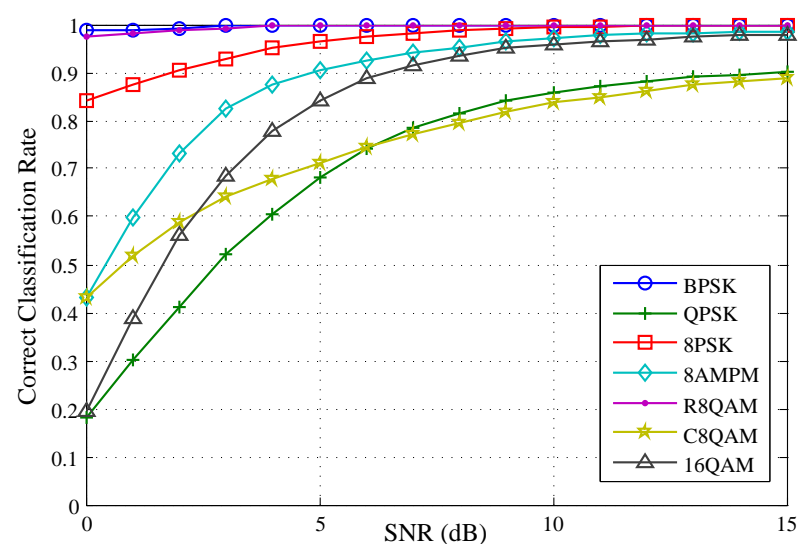

Fig. 5. Correct Classification Rate as a function of SNR

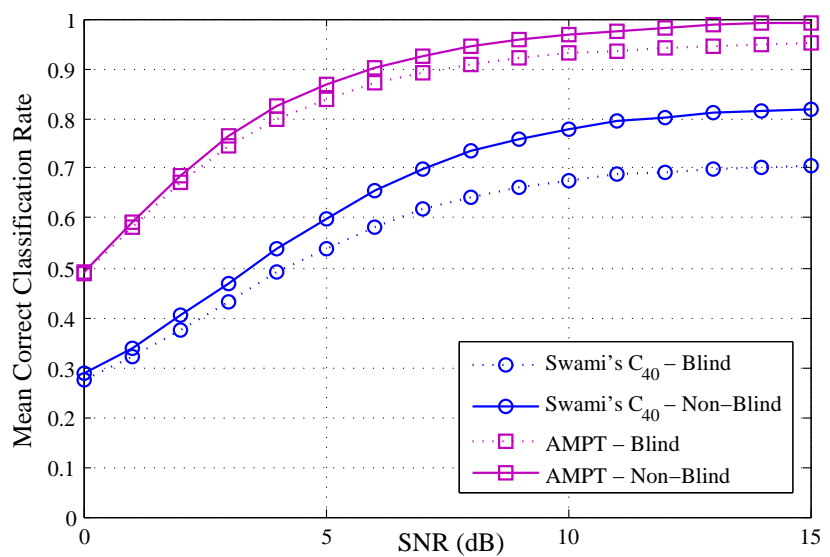

Fig. 6. Mean CCR for both methods in the blind/non-blind scenario

\subsection{Complexity comparison with the $C_{40}$-based classifier}

Last, we compare the $2 \mathrm{PT} / 4 \mathrm{PT}$-based method with the $C_{40}$-based classifier in terms of complexity. In both cases, the greediest operation is the computation of the features.

The computation of $M \mathrm{PT}\left(M \widehat{f}_{r}\right)(2)$ asymptotically requires $M N_{e}$ complex multiplications and $N_{e}$ complex additions if the exponential was precomputed. Then, the 2PT/4PT-based classifier requires $6 N_{e}$ complex multiplications and $2 N_{e}$ complex additions. In comparison, the computation of $\widehat{C}_{40}$ is slightly lower since it requires $4 N_{e}$ complex multiplications and $2 N_{e}$ complex additions.

\section{CONCLUSION}

Throughout this article, a new efficient way to blindly classify digital modulations is described. The method is based on an Analytical study of the $M^{t h}$-Power nonlinear Transformation. The featurevector used for the classification is insensitive to time, phase and frequency offsets, which makes the whole classifier more robust to estimation uncertainty in the blind scenario.

The key features of the proposed method are the absence of complex preprocessing and its low complexity. These properties make it a great tool in smart receivers for wide-band monitoring and for the estimation of the features of the signal before the demodulation step.

Further work shall be carried out, for instance regarding the statistical distribution of the feature-vector in realistic conditions. The effects of multi-path channels on the analytical development of the references shall also be investigated. 


\section{REFERENCES}

[1] E. Axell, G. Leus, E.G. Larsson, and H.V. Poor, "Spectrum Sensing for Cognitive Radio: State-of-the-Art and Recent Advances," IEEE Sig. Proc. Magazine, vol. 3, no. 29, 2012.

[2] Jacques Palicot and Christian Roland, "A New Concept for Wireless Reconfigurable Receivers," IEEE Communications Magazine, vol. 41, no. 7, 2003.

[3] Z. Khalaf, A. Nafkha, and J. Palicot, "Blind Cyclostationary Feature Detector based on Sparsity Hypotheses for Cognitive Radio Equipment," IEEE International Midwest Symposium on Circuits and Systems, 2011.

[4] Octavia A. Dobre, A. Abdi, Y. Bar-Ness, and Wei Su, "Survey of Automatic Modulation Classification Techniques: Classical Approaches and New Trends," IET Communications, vol. 1, no. 2, pp. 137-156, 2007.

[5] Alharbi Hazza, Mobien Shoaib, Saleh A. Alshebeili, and Alturki Fahad, "An Overview of Feature-based Methods for Digital Modulation Classification," International Conference on Communications, Signal Processing, and their Applications, vol. 1, no. 08, pp. 1-6, 2013.

[6] Cheol-Sun Park and Dae Young Kim, "A Novel Robust Feature of Modulation Classification for Reconfigurable Software Radio," IEEE Transactions on Consumer Electronics, vol. 52, pp. 1193-1200, 2006.

[7] A. Swami and B. M. Sadler, "Hierarchical Digital Modulation Classification using Cumulants," IEEE Transactions on Communications, vol. 48, no. 3, pp. 416-429, 2000.

[8] Wei Dai, Youzheng Wang, and Jing Wang, "Joint Power Estimation and Modulation Classification Using Second-and Higher Statistics," Wireless Communications and Networking Conference (WCNC), pp. 155-158, 2002.

[9] V. Chaithanya and Vellenki Umapathi Reddy, "Blind Modulation Classification in the Presence of Carrier Frequency Offset," International Conference on Signal Processing and Communications, pp. 1-5, 2010.

[10] Markus Flohberger, Wilfried Gappmair, and Otto Koudelka, "Modulation Classifier for Signals used in Satellite Communications," Advanced Satellite Multimedia Systems Conference and Signal Processing for Space Communications Workshop, pp. 198-202, 2010.

[11] Octavia A. Dobre, A. Abdi, Y. Bar-Ness, and Wei Su, "Cyclostationarity-Based Blind Classification of Analog and Digital Modulations," IEEE Military Communications Conference (MILCOM), pp. 1-7, 2006.

[12] Fangjuan Xie, Chisheng Li, and Guojin Wan, "An Efficient and Simple Method of MPSK Modulation Classification," International Conference on Wireless Communications, Networking and Mobile Computing, , no. 1, pp. 1-3, 2008.

[13] Chia Wei Lim and Michael B. Wakin, "Automatic Modulation Recognition for Spectrum Sensing using Nonuniform Compressive Samples," IEEE International Conference on Communications (ICC), 2012.

[14] Zhengli Xing et al., "Automatic Modulation Recognition of PSK Signals using Nonuniform Compressive Samples based on HOS," IEEE International Conference on Communication Problem-Solving, 2014.

[15] JE Mazo, "Jitter Comparison of Tones Generated by Squaring and by Fourth-Power Circuits," Bell System Journal, 1978.

[16] J. Reichert, "Automatic Classification of Communication Signals using Higher Order Statistics," IEEE International Conference on Acoustics, Speech, and Signal Processing, vol. 5, no. 4, pp. 221-224, 1992. 